\title{
Adjustments of public transit operations planning process for the use of electric buses
}

Carl Henrik Häll, Avishai (Avi) Ceder, J oakim Ekström and Nils-Hassan Quttineh

The self-archived postprint version of this journal article is available at Linköping University Institutional Repository (DiVA):

http:// urn.kb.se/ resolve?urn=urn:nbn:se:liu:diva-152547

N.B.: When citing this work, cite the original publication.

This is an electronic version of an article published in:

Häll, C. H., Ceder, A. (., Ekström, J., Quttineh, N., (2018), Adjustments of public transit operations planning process for the use of electric buses, J ournal of Intelligent Transportation Systems / Taylor \& Francis. https:// doi.org/ 10.1080/ 15472450.2018.1488131

Original publication available at:

https:// doi.org/ 10.1080/15472450.2018.1488131

Copyright: Taylor \& Francis (STM, Behavioural Science and Public Health Titles)

http:// www.tandf.co.uk/journals/ default.asp 


\title{
Adjustments of Public Transit Operations Planning Process for the Use of Electric Buses
}

\author{
Carl H. Häll* Avishai (Avi) Ceder ${ }^{\dagger} \quad$ Joakim Ekström* \\ Nils-Hassan Quttineh ${ }^{\ddagger}$
}

\begin{abstract}
This work investigates and discusses how the introduction of electric buses (EB), both battery and plug-in hybrid EB, will and should change the operations planning of a public transit system. It is shown that some changes are required in the design of a transit route network, and in the timetabling and vehicle scheduling processes. Other changes are not required, but are advisable, using this opportunity upon the introduction of EB. The work covers the main characteristics of different types of EB with a short description, including the most popular charging technologies, and it presents the generally accepted transit operations planning process. Likewise, it describes and analytically formulates new challenges that arise when introducing EB. The outcome of the analyses shows that multiple new considerations must take place. It is also shown that the different charging techniques will influence the operations planning process in different ways and to a varying extent. With overnight, quick and continuous charging, the main challenges are in the network route design step, given the possibility of altering the existing network of routes, with efficient and optimal changes of the timetabling and vehicle scheduling components. An illustrative example, based on four bus lines in Norrköping, Sweden, is formulized and introduced using three problem instances of 48, 82 and 116 bus trips. The main results exhibit the minimum number of vehicles required using different scenarios of charging stations.
\end{abstract}

Keywords: electric buses; transit operations planning process; charging technologies; optimal route design; optimal scheduling

*Department of Science and Technology, Linköping University, Sweden

${ }^{\dagger}$ Corresponding author; Department of Civil and Environmental Engineering, University of Auckland, New Zealand, and International Development and Cooperation (IDEC), Hiroshima University, Japan. E-MAIL: a.ceder@auckland.ac.nz.

¥Department of Mathematics, Linköping University, Sweden 


\section{Introduction}

Electric buses, based on either battery or hybrid technologies, are currently being introduced in public transit systems all over the world. The benefits of operating electric buses are many, with the two main arguments being that electric buses produce no local emissions and they operate under very low noise levels. While these arguments hold true for the case of battery electric buses, they are subjected to the operating conditions and charging infrastructure for the case of plug-in hybrid electric buses. It is likely that various types of electric buses will become more and more common in cities around the world. According to ZeEUS (2017) the global sales of electric buses is expected to grow by $33.5 \%$ annually during the period 2017 $-2025$.

Compared to conventional buses (buses with traditional combustion engines), the introduction of electric buses brings new complexities to the public transit planning process. Charging infrastructure needs to be considered, and additional technological choices need to be made. By virtue of their introduction not only is the opportunity presented to substitute buses and provide charging infrastructure, but to a large extent this is an opportunity to redesign the public transit system. Major changes to a transit system are not easy to implement. Among other things, this is because changes are often not appreciated if it is not immediately quite clear that they result in an improvement. Therefore, introduction of new technology, such as electric buses and charging infrastructure, that represents a clear improvement to the public provides a favorable opportunity for introducing other changes to the system.

In this paper we investigate and discuss how the introduction of electric buses, both battery and plug-in hybrid electric buses, will and should change the planning of a public transit system. Some changes are necessary to introduce into the planning process when introducing electric buses, while other changes may be beneficial to introduce at the same time. While discussing these topics, we also provide a literature review of previous work on the different steps in the planning process in relation to the introduction of electric buses. This work is part of a research project funded by the Swedish Energy Agency, and we have collaborated with a group of Swedish practitioners (local municipalities, public transit providers and operators). The companies have supported us as a reference group, and provided us with data and practitioners perspectives.

The paper is organized in the following way: Section 2 gives a short description of the main characteristics of different types of electric buses and the most popular charging technologies. Section 3 presents the generally accepted operations planning process for public transit, and in Section 4 we describe the new challenges that arise (in each of the planning steps) when introducing electric buses, and how the previous

literature has addressed them. Section 5 summarizes our findings and proposes a new planning process, adapted to the conditions and requirements of electric buses. Section 6 illustrates a numerical real-life example of how the vehicle scheduling step 
can be impacted by the introducing of electric buses. Conclusions are presented in Section 7.

\section{Technologies for electrification of bus routes}

Over the course of history, electric powered buses have emerged and disappeared in public transit systems around the world. Electric buses can be traced back to the Siemens trolleybus in 1882, and an early adoption was Aberdeen in UK 1909 (Brunton, 1992). Since then, trolley buses have become a part of the public transit system in many cities. Today there is a renewed interest in electrified buses, but this time without the need of ever-present infrastructure for the delivery of electricity to the buses, thus allowing cheaper and more flexible deployment.

Electric powered buses exist in a wider range of designs than conventional buses. While both electric powered buses and conventional buses come in different sizes, the electric powered buses also vary in terms of battery size and engine technique. The two main types of electric buses, which are currently being considered and tested in public transit systems today, are battery electric buses and plug-in hybrid electric buses. Within these categories there are a large variety of technological approaches, but here we will focus primarily on battery size and alternative charging techniques. A large battery is heavy and takes up more space, thus it will increase the power needed to propulse the bus, and reduce the number of maximum passengers on the bus. A smaller battery will instead reduce the power needed and increase the number of passengers, but on the other hand reduce the range. Thus, the choice of bus is heavily dependent on the design of the bus lines and the charging infrastructure, a dependency which is of less importance for conventional buses. J.-Q. Li (2016) gives an up-to-date overview of current deployment of heavy-duty electric buses (of approximately 11 to 12 meters in length) around the world.

A review on alternative powertrains for electric buses are presented by Mahmoud, Garnett, Ferguson, and Kanaroglou (2016) and Mohamed, Farag, El-Taweel, and Ferguson (2017). These review articles provide a holistic assessment of electric buses by comparing different powertrains from economic, operational, energy, and environmental perspectives. This holistic assessment is based on 16 different performance features and concludes that several perspectives should be considered when deciding which charging technology and vehicle technique to use.

\subsection{Charging technology}

Independent of whether the buses considered are battery or plug-in hybrid electric buses, there is a need to charge them. Five main approaches can be distinguished in the literature, e.g., see J.-Q. Li (2016) and Olsson, Grauers, and Pettersson (2016):

- Charging at a depot can either be done overnight, or by having the bus return to the depot for charging during the day, in the case of a bus only running during 
the morning and afternoon peaks. For a battery electric bus, the battery size needs to be large enough for the bus to operate a whole day or part of a day.

- Opportunity charging at end points can be used for charging a bus during a longer pause, before continuing to its next trip. The battery size needs to be large enough for the bus to occasionally skip an endpoint charging if there are delays. If the bus leaves the depot with a fully charged battery, it can return with a low charged battery in the evening, thus the opportunity charging can be planned so as to not fully repower the batteries during the day.

- Opportunity charging at bus stops can be used for rapid charging during operations. It can either be used as the main source of charging, with chargings at several stops along a line. Alternatively, it can be used as a reserve charging facility, if the bus has missed previous charging opportunities at end points.

- Charging during driving can be done with several technologies, for example overhead lines or inductive energy transfer beneath the tarmak. In a hybrid electric bus this may also be done by charging from an internal combustion engine.

- Battery swapping can be used for extending the range of a battery electric bus. The onboard energy storage is then completely restored after the battery swap. According to J.-Q. Li (2016) the main problem with battery swapping is that it requires large areas for the battery swapping stations, which are also costly to build.

Depending on the charging technique used, the battery size and charging power will differ. Charging a bus at a depot requires less charging power since the charging is performed over a longer time, but on the other hand, large batteries are also required for the bus to operate a long time period without charging. Rapid opportunity charging at bus stops on the other hand requires charging power that is high enough to charge the battery over a short time period (30-60 seconds), but the batteries can be smaller.

The battery size for charging during driving depends on the size of the part of the route being electrified. Olsson et al. (2016) develop a model which can determine the optimal combination of battery size and charging infrastructure depending on costs associated with both charging infrastructure, vehicles and batteries. Their results highlight the need for optimizing vehicle technology, battery size and charging infrastructure at a system level, taking the operational aspects into account. While overnight charges require less power (about $80 \mathrm{kWh}$ per charge) (Olsson et al., 2016), many buses will be charged at the same location. Therefore, the impact on the electricity grid from the overnight chargers will be considerable. This impact is investigated using simulation for both overnight charging and other charging technologies by Mohamed et al. (2017). For a full transit network (comprised of 9 
lines) this study creates load profiles, for different electric bus configurations, and analyze the impacts on the local distribution grid.

\subsection{Vehicle techniques}

There are two main types of electric buses - battery electric buses and plug-in, hybrid electric buses. What characterize, and differentiates, them are described in the following two subsections.

\subsubsection{Battery electric buses}

Battery electric buses rely solely on an on-board battery to deliver electricity for the engine. Thus, the operational range is dependent on the size of the battery, in terms of kWh. Battery size required for operating the bus in a public transit system also depends on the available charging infrastructure.

Battery electric buses have the benefit of having zero tailpipe emission, but on the other hand they rely on a large enough battery. Thus, depending on the battery size, battery electric buses are available with different maximum ranges. For example, the Chinese electric bus, BYD K9, has been tested in Copenhagen, Denmark, with only overnight charging, showing a maximum range of $325 \mathrm{~km}$ in light traffic and $250 \mathrm{~km}$ in heavy traffic (Hug, 2015).

\subsubsection{Hybrid electric buses}

Hybrid electric buses combine a combustion engine with an electric motor. This can either be done in parallel or in series. In a parallel set-up both types of motors can be used for propulsion. In contrast, the series set-up includes one electric motor, used for propulsion, and one combustion engine as a generator, used for providing electricity to the electric motor, when it is not running on electricity from a battery. The two approaches can be combined in a series-parallel set-up, which can take advantage of the benefits from both series and parallel set-ups. For an overview of hybrid bus architectures, we refer to the work of Živanović and Nikolić (2012).

Hybrid engines are subjected to powertrain control, which based on some energy management strategy determines when and how the two different engines are operated. The powertrain control design has a large impact on the engine performance, energy use and emissions. Bayindir, Gözüküçük, and Teke (2011) list four main goals with the powertrain control: maximum fuel economy, minimum emissions, minimum system cost and good driving performance.

For hybrid buses with the capability of running only on the electric engine, the operator (or ultimately the authorities) may want to restrict when and where the bus can use the combustion engine. Thus creating areas of zero emission and noise. This can be achieved by geo-fencing, but it should be noted that if such a management strategy overrides manufacturer powertrain control strategies, the engine performance, in terms of energy use and emissions, may not be as promised 
nor as used when determining legislative emissions classes. This could, for instance, happen if the combustion engine is running only for short intervals, never reaching optimal operating temperature. This conflict of interests is apparent, but not well considered in the literature, which mainly focuses on powertrain management from the manufacturer perspective.

\subsection{Planning aspects}

The deployment of electric vehicles requires charging infrastructure. The location of this infrastructure, and choice of charging technology, does not only have an impact on the transport system, but also on the electricity grid. Nowadays the implementation of electric vehicles is discussed and developed for private cars such as the recent interesting study of Xu, Çolak, Kara, Moura, and González (2018). However, this line of research is quite complex with uncertain parameters dependent on individual users. This work focuses on public transport vehicles, especially buses, where the problem can be structured with less uncertainties compared with the private cars.

As pointed out in Olsson et al. (2016), the charging infrastructure cost may not be a determining cost for choosing vehicles and battery size, and designing the charging system. Thus, there is a need to consider other aspects of the public transit planning as well. For instance, using opportunity charging, either at bus stops or end points, is related to the design of the timetables, and the possibility of installing charging stations will influence the network route design.

In comparison to conventional buses, battery electric buses are less flexible, since their operation depends on the availability of charging infrastructure. The battery charging also introduces additional complexity to everyday operations, as battery electric buses need to be recharged regularly, independent of whether the bus is delayed for some reason. Also, the planning must allow for alternative routes during road closure, and future expansions and changes of the route network. Thus, there is a need for robust planning of the networks, timetables, vehicles and charging infrastructure.

\section{The public transit operations planning process}

The classical approach to Transit Operations Planning is to decompose the planning process into several steps, usually network route design (NRD), timetable development (TD), vehicle scheduling (VS) and crew scheduling (CS). This section presents a short introduction to these planning steps. For a more detailed description see Ceder (2016).

We start by mapping inputs and outputs for the traditional decision problems, as well as dependencies between planning steps. An illustration of the planning 


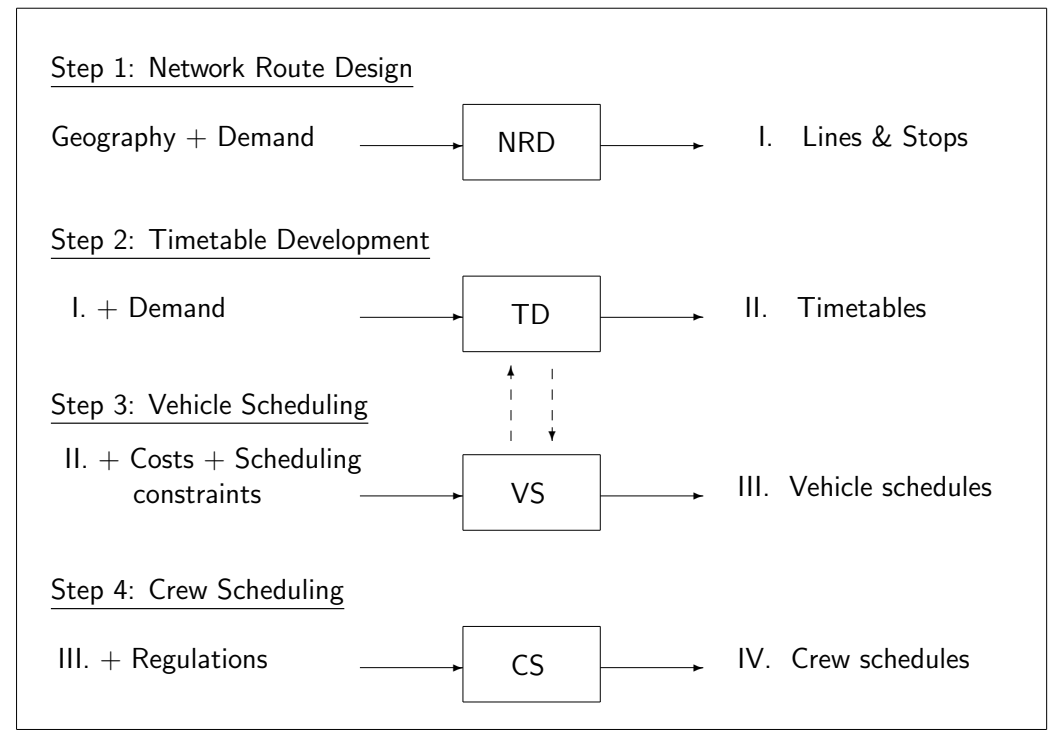

Figure 1: Overview of the transit operations planning process.

process is given in Figure 1. This planning process takes many perspectives into account; for example, passengers, public transit agencies and communities (local and/or national government).

In the first step, the bus lines and locations of stops are designed based on estimated travel demands and geographical information regarding existing infrastructure, traffic volumes on the road network (travel speeds), populations in different regions, etc. The placement of the bus depot (or several depots) can also be part of this planning step. Output from the network route design is a set of bus lines and stops. When formulated as an optimization problem, this step is referred to as the network design problem (NDP).

In the second step, frequencies (and headways) of the different lines are first set. Timetables that fulfill these frequencies are subsequently constructed. For this reason, frequency setting and timetable construction can sometimes be referred to as two separate steps. This step is solved based on the output from the NRD (lines+stops) and travel demands. Output is the timetables and frequencies for all lines. The corresponding optimization problem is referred to as the bus timetabling problem (BTP).

The third step is to create feasible vehicle schedules for the fleet of vehicles, based on the timetables created in the previous step together with cost factors and scheduling constraints. The corresponding optimization problem is known as the Vehicle Scheduling Problem (VSP). However, the timetable development (TD) and the vehicle scheduling (VS) steps are often iterated, to evaluate how changes in the original timetables affect the vehicle scheduling. Most often such changes are created by shifting departure times in order to utilize the transit agency's resources (vehicles and crew) as efficiently as possible. Such, possible small, changes can give 
rise to new ways to create efficient interlining.

In the last planning step, one should assign which crew member (bus driver) should perform which duty (part of a vehicle schedule). This is known as the crewscheduling problem (CSP). This step is, of course, based on the output from the VS step, because it gives information about existing duties, together with workhour regulations. Many bus transit planning and scheduling models and solution algorithms, including exact algorithms, heuristic algorithms and metaheuristic algorithms, have been developed to solve bus transit vehicle and crew (driver) scheduling/rostering problems. For detailed summaries and reviews of these models and algorithms, readers are referred to Ceder (2016) and Ibarra-Rojas, Delgado, Giesen, and Muñoz (2015).

These four steps describe the overall planning process. However, one should bear in mind that it is very rare that a city completely redesigns its transit network. Most often only small changes, if any, are made to the first planning step. When introducing electric buses, the introduction can be made with either very small changes to the network, or at the same time taking the opportunity to introduce other network changes. In the upcoming section, we describe how the deployment of electric and hybrid buses will affect the planning process with respect to different situations; quick charging, overnight charging and continuous charging.

\section{Effects of introducing electric buses}

With respect to public transit planning, we group the charging approaches described earlier into three categories:

- Quick charging: This category consists of any technology which will allow recharging within a few minutes, and hence includes opportunity charging (both at end points and at bus stops) and battery swapping.

- Overnight charging: Here we consider any technology which requires charging times of 30 minutes or more (up to several hours). We assume that a fully charged battery will allow the bus to operate for a full day (charging at a depot).

- Continuous charging: This category covers all technologies that require a continuous charging, such as overhead lines (charging during driving).

Based on these categories, we will now analyze each of the planning steps mentioned in the previous section and describe how they are affected by the different charging technologies. As will be shown, all planning steps are not affected by all technologies, and for guidance and support for the reader we summarize our findings in Table 1.

In the upcoming subsections, one for each planning step, we provide a literature overview, followed by an analysis of how the different charging categories will affect the planning step (if at all). 
Table 1: Overview of different charging techniques for electric buses and their influence on the public transit operations planning process (no influence for empty cell).

\begin{tabular}{|l|c|c|c|}
\hline & $\begin{array}{c}\text { Overnight } \\
\text { charging }\end{array}$ & $\begin{array}{c}\text { Quick } \\
\text { charging }\end{array}$ & $\begin{array}{c}\text { Continuous } \\
\text { charging }\end{array}$ \\
\hline Network route design & $\checkmark$ & $\checkmark$ & $\checkmark$ \\
\hline Timetable development & & $\checkmark$ & \\
\hline Vehicle scheduling & $\checkmark$ & $\checkmark$ & \\
\hline Crew scheduling & & & \\
\hline
\end{tabular}

\subsection{Network route design (NRD)}

When introducing electric buses to a transit system, it is not likely that the entire bus fleet will be exchanged at once. It is more reasonable to believe that a few electric buses at a time are introduced into an existing fleet of vehicles. Beltran, Carrese, Cipriani, and Petrelli (2009) describe how a transit network design problem for which only part of the vehicle fleet is considered as green vehicles (for example electric vehicles) can be tackled. For this network design problem, a large set of possible routes are generated and a genetic algorithm is then used to find the optimal subset of these routes.

The questions of where to locate charging stations and what their dimensions should be, are of course important to consider during the network design. These problems are discussed in the work of Zhu, Chen, and Xie (2013) regarding battery swap stations. Two objectives are considered; construction cost and closeness for all lines from the end point of the line to a charging station, and four different sizes (capacities) of charging stations can be used. The charging demand at each end point of the lines were assumed to be known. The demand at different charging stations however depend heavily on the characteristics (for example geography and demand variations) of the bus routes that are using the charging station. In Perrotta et al. (2014) simulations are performed to study the energy usage for different types of bus routes. Three routes from the city of Oporto are used in the simulations that are based on both nanoscopic description of the electric buses and microscopic interaction with other traffic.

\subsubsection{Overnight charging}

With overnight charging, the localization of the bus depot will be essential, as it will need access to a high-voltage/high-capacity electricity grid. The location also affects deadheadings to and from the lines start- and end stops.

The cost for building a depot can vary between locations. Thus a tradeoff scenario may exist by a highly suitable location, but with a high building cost. The 
possibility to upgrade existing depots with a charging infrastructure should also be considered and evaluated. Even though building, or upgrading, depots may be costly, it may prove preferable, or even necessary, to build more than one depot. Thus, an additional question for the NRD step to address, relates to the additional output to be generated, in the case of overnight charging, as a means of determining the locations and numbers of depots to be built.

\subsubsection{Quick charging}

With a fleet of electrical buses using quick charging, a need for new input to the NRD step is introduced. It is always part of the NRD to decide the locations of bus stops, but now it also includes determining the locations for charging stations. In most practical cases with quick charging, such locations will be installed at a subset of the bus stops.

The NRD step must therefore be modified to also identify possible, and/or suitable, bus stops where quick charging could be performed/installed. To get this information from the NRD step it is necessary to clearly define the criteria that constitutes a good location for charging. These criteria are used as new input to the NRD step. The output from the NRD step is still the lines and stops of the network, but now it contains a set of possible charging stops as well.

The layout of the lines in the network will naturally affect which stops are suitable for charging. However, the effect can also be in the opposite direction the choice of charging locations may affect the layout of the lines. This is due to the fact that allowing several lines to use the same charging infrastructure can be an efficient solution.

\subsubsection{Continuous charging}

It is likely that continuous charging will affect the layout of the lines in the network. As mentioned for quick charging, it may be a good solution if several lines can use the same charging infrastructure. In the case of continuous charging, the cost of electrification is directly dependent on the distance of road to be electrified. For this reason, it could very well become more advantageous to build dedicated bus lanes or separate bus streets (where the technology is installed) to be shared for multiple bus lines.

If the vehicles only require electrification on part of the roadway, then the planning of networks for such vehicles also gives rise to an additional problem: choosing a subset of links where the technology needs to be installed so that all buses get sufficient charging in order to run all day long, with the objective of minimizing installation costs. 


\subsection{Timetable development (TD)}

There is vast literature on Public Transport Timetabling, and a majority of it deals with bus timetabling. The most common objective seems to be minimization of passenger waiting times, or synchronization of bus line departures. In addition the matter of robustness is important to consider in the creation of timetables. Corman, D'Ariano, and Hansen (2014) discusses the questions of what is a robust timetable, and how robustness can be quantified. If the answers to these questions are clear then this can be included in the objective of the timetable development (TD) step.

In Ceder, Golany, and Tal (2001), a mixed-integer programming (MIP) model for creating bus timetables with maximal synchronization is given, and an efficient heuristic approach is proposed. In Liu, Shen, Wang, and Yang (2007), a Nesting Tabu Search algorithm is presented which aims to maximize the number of synchronized departures. In the more recent Ibarra-Rojas and Rios-Solis (2012), another MIP-model is given for maximization of synchronizations in the bus system in Mexico, and Saharidis, Dimitropoulos, and Skordilis (2014) gives a MIP-model for minimization of waiting times in the public bus transportation system in Greece.

Another recent paper by Parbo, Nielsen, and Prato (2014), shows the social benefits of an efficient public transportation system. Here a tabu search heuristic was applied to the public transport system of Denmark, yielding a whopping 45 million Danish Kroner (DKK) savings due to reduced waiting times.

To the best of our knowledge, there are no published papers specifically dealing with timetabling of electric buses. This is reasonable since neither the technology of overnight charging, nor that of continuous charging, will have any significant effects on the timetable development. However, for a bus fleet using quick charging, the timetabling problem will indeed be affected. Exactly how this occurs is explained in the following section.

The creation of timetables can be done in various ways (with different purposes/objectives). For example, one might request even headways on lines or even demand loads on vehicles (departures). We have chosen to exemplify the creation of timetables with the case of maximum synchronization. (For a more detailed description of the model, we refer to Ceder (2016) and Ceder et al. (2001)). The problem of creating a timetable (setting departure times) with as many synchronized stops as possible can be formulated as

$$
\max \sum_{k=1}^{M-1} \sum_{i=1}^{F_{k}} \sum_{q=k+1}^{M} \sum_{j=1}^{F_{q}} \sum_{n=1}^{N} z_{i k j q n}
$$

subject to 


$$
\begin{array}{rlrl}
x_{1 k} & \leq \operatorname{Hmax}_{k} & k=1, \ldots, M \\
x_{F_{k}, k} & \leq T & k=1, \ldots, M & \\
x_{i+1, k}-x_{i k} & \geq \operatorname{Hmin}_{k} & k=1, \ldots, M, i=1, \ldots, F_{k}-1 \\
x_{i+1, k}-x_{i k} & \leq \operatorname{Hmax}_{k} \quad k=1, \ldots, M, i=1, \ldots, F_{k}-1 \\
z_{i k j q n}=\max & \left\{1-\left|\left(x_{i k}+T_{k n}\right)-\left(x_{j q}+T_{q n}\right)\right|, 0\right\} \\
k, q & =1, \ldots, M, i=1, \ldots, F_{k}, j=1, \ldots, F_{q}, n=1, \ldots, N
\end{array}
$$

where:

$x_{i k}$ is a variable specifying the $i$ th departure time on route $k$.

$z_{i k j q n}$ is a binary variable that takes value 1 if the vehicle of the $i$ th departure on route $k$ meets the vehicle of the $j$ th departure of route $q$ at node $n$; and 0 otherwise. $M$ is the number of routes.

$N$ is the number of transfer nodes.

$F_{k}$ is the number of departures at route $k$.

$T$ is the planning horizon.

$T_{k j}$ is the travel time from start node to node $j$ on route $k$.

$\mathrm{Hmin}_{k}$ is the minimum headway between two departures on route $k$.

$\operatorname{Hmax}_{k}$ is the maximum headway between two departures on route $k$.

In this formulation, the objective function (1) maximizes the number of coordinated stops at transfer nodes. Constraint (2) ensures that the first departure of route $k$ is not scheduled later than the maximum headway time after the start of the planning horizon, and constraint (3) makes sure that the last departure of route $k$ is scheduled no later than the end of the planning horizon. Constraints (4) and (5) checks that the headway limits are met, and constraint (6) sets the binary variables for the objective function.

\subsubsection{Quick charging}

As before, synchronization aspects should be part of the model, but now with respect to charging as well. If a bus needs to wait a few minutes for a connecting bus, it may as well use the time to charge its batteries.

In order to incorporate this into the timetables, charging time parameters must be provided as input to the TD step. Depending on the technology, a quick charge could take from 30 seconds to several minutes, which will affect the timetabling. In the former case, the charging could be performed while passengers embark and exit the bus, while in the latter case, the charging must be performed at certain stops where it waits for a connecting line. The latter also introduces some restrictions with respect to space, since the bus cannot block the other traffic. 
If it is known from the NRD-step where the charging infrastructure is available, a new set of parameters $c_{n}$ can be introduced. Charging infrastructure installed at node $n$ is described as:

$$
c_{n}= \begin{cases}\alpha & \text { if charging is available at node } n \\ 1 & \text { otherwise }\end{cases}
$$

where the value $\alpha$ indicates the weight of having a synchronized transfer occurring at a node where charging is available.

Based on the assumption that a synchronized meeting takes a bit longer on the average than the time spent at a regular bus stop, it is reasonable to say that it would be beneficial to use that time for charging. If it is also assumed that charging can be made during one unit of time (basically the time limit for what is considered as a synchronized meeting) then the objective function can simply be changed to:

$$
\max \sum_{k=1}^{M-1} \sum_{i=1}^{F_{k}} \sum_{q=k+1}^{M} \sum_{j=1}^{F_{q}} \sum_{n=1}^{N} c_{n} z_{i k j q n}
$$

If charging locations are not given from the NRD-step, one must treat $c_{n}$ as variables, indicating if charging infrastructure should be installed. In such a case, constraints must also be added to regulate the number of locations at which charging can be provided. These simple changes to prioritize synchronizations at nodes with charging infrastructure can of course be extended to allow charging at other (e.g., end point) locations and with more detailed information regarding different charging times for different buses, etc.

The output from TD step is obviously still timetables. However, in addition, it should now include information about when and where charging should take place, as well as where charging infrastructure should be installed.

\subsection{Vehicle Scheduling (VS)}

The vehicle scheduling problem for electric buses has been tackled in different ways in the literature in the past, both with exact models and with heuristics for different electrification technologies. One of the early papers that models the electric bus scheduling problem is that of Wang and Shen (2007), in which the problem is modeled as a vehicle scheduling problem with route and fueling time constraints. This problem is solved using an ant colony algorithm.

Zhu and Chen (2013) model a vehicle scheduling problem for a fleet of electric vehicles using battery swap technique. The problem is formulated as a single depot vehicle scheduling problem with route time constraints. The authors consider two objectives: cost of vehicles and extra batteries, and cost of charging. For a case study with data from Shanghai, Pareto optimal solutions are presented. The interesting thing about this is the fact that some solutions to the vehicle scheduling problem 
generate a need for a larger number of extra batteries in the system, and/or a larger demand for charging. For this reason it is important to weigh the costs of charging against that of the capital investment in batteries.

J. Li (2014) presents a vehicle scheduling model for battery swapping or fast charging at battery stations. The author claims that the time required for battery swapping is similar to that of fast charging and that the same model can be used for both techniques. Algorithms based on column generation are proposed to solve the scheduling problems. Both real world instances and randomly generated instances are used to evaluate the algorithms.

Reuer, Kliewer, and Wolbeck (2015) tackles a similar problem which extends a traditional vehicle scheduling problem to consider limited battery capacity and vehicle recharging options. They define the electric vehicle scheduling problem (EVSP) as an extension of a standard vehicle scheduling problem (VSP), and a multivehicle type vehicle scheduling problem (MVT-VSP), as well as for electric vehicles to be considered in terms of a multivehicle type electric vehicle scheduling problem (MVT-EVSP). The extension for electrical vehicles is that the model must ensure that batteries are not depleted, while vehicles can only charge at predefined stops. The argumentation for the need of a multivehicle type formulation is that the introduction of electric buses will most likely progress over the years, and that during some of those years many fleets will have a mix of different vehicle types. An existing time-space network based exact solution approach for VSP (Kliewer, Mellouli, \& Suhl, 2006) is used and an algorithm is presented that adds charging when necessary. Different strategies for network flow decomposition are also tested.

The purpose of the VS-step is in general to minimize the number of vehicles needed to carry out the timetables. To illustrate as simply as possible how this can be done and how it can be affected by the introduction of electric buses, we use an example of a Multi-Depot Vehicle Scheduling Problem (MDVSP). In the first stage of solving a MDVSP, trip chains are generated. The second stage is to assign buses from different depots to each chain. For our purposes, the first stage is the most interesting to illustrate, since when introducing electric buses one needs to make sure that there are sufficient charging possibilities in each chain. For more detailed information about the MDVSP we refer to Ceder (2016).

The chaining of trips can be modeled in the following way:

$$
\min \sum_{i=1}^{n+1} \sum_{j=1}^{n+1} c_{i j} y_{i j}
$$

subject to 


$$
\begin{array}{lll}
\sum_{i=1}^{n+1} y_{i j}=1 & j=1, \ldots, n \\
\sum_{j=1}^{n+1} y_{i j}=1 & i=1, \ldots, n \\
\sum_{j=1}^{n+1} y_{n+1, j}=n & i=1, \ldots, n \\
\sum_{i=1}^{n+1} y_{i, n+1}=n & j=1, \ldots, n
\end{array}
$$

In addition to these constraints, there are also constraints ensuring that trip end $i$ can link to trip start $j$, and that $y_{i j}$ are integers and greater than or equal to zero. In the model, there are $n$ trip events and it is assumed that we start with a fleet of $n$ buses. The variables are defined as follows:

$$
\begin{aligned}
& y_{i j}= \begin{cases}1 & \text { if trip end } i \text { is linked to trip start } j \quad i, j=1, \ldots, n \\
0 & \text { otherwise }\end{cases} \\
& y_{n+1, j}=\left\{\begin{array}{ll}
1 & \text { if depot supplies trip start } j \\
0 & \text { otherwise }
\end{array} \quad j=1, \ldots, n\right. \\
& y_{i, n+1}=\left\{\begin{array}{ll}
1 & \text { if depot supplies trip start } j \\
0 & \text { otherwise }
\end{array} \quad i=1, \ldots, n\right. \\
& y_{n+1, n+1}=\text { number of buses unused }
\end{aligned}
$$

and the costs, $c_{i j}$, are the following:

$$
c_{i j}= \begin{cases}V & i=n+1, \quad j=1, \ldots, n \\ 0 & i=1, \ldots, n, \quad j=1, \ldots, n+1 \\ L_{i j}+E_{i j} & i=1, \ldots, n, \quad j=1, \ldots, n\end{cases}
$$

where $V$ is the cost of using a vehicle, $L_{i j}$ is the deadheading cost from trip end $i$ to trip start $j$ and $E_{i j}$ is the idle time cost between $i$ and $j$.

In the described model, the objective function (8) minimizes the cost of the trip chains created. Constraints (9) and (10) guarantee that exactly one vehicle arrives at each trips start node, and that exactly one vehicle leaves each trips end node. Constraints (11) and (12) make sure that all vehicles leave and return to the depot.

The following subsections will describe a simple way of changing this model to be useful for planning vehicle schedules for electric buses using overnight charging and quick charging. In the case of continuous charging, the vehicle scheduling (VS) step is not affected. 


\subsubsection{Overnight charging}

If the buses use overnight charging, we can either simply assume that they are able to run all day without any restrictions on routes, or specifications are applied to the bus fleet (for example number of buses of each type, distance restrictions for each type, etc.) as input to the VS step.

In the simplest of cases, where a homogeneous fleet of vehicles is assumed, for overnight charging one can simply extend the original model with constraints making sure that each trip chain created can be served by a bus with the specifications of the bus type used. For example, the total power consumption for the bus to perform the trip chain is less than the battery can store. However, the easiest way to do so and to illustrate what happens is to reformulate the problem to keep track of which vehicle is taking which chain of trips. This means that the problem is reformulated to a MultiDepot Vehicle Scheduling Problem with Route Time Constraints (MDVSRTC). The most important changes are that vehicle specific variables $y_{i j k}$ are introduced and the addition of the route time constraints, here considered as power consumption constraints. The model can now be formulated as:

$$
\min \sum_{i=1}^{n+1} \sum_{j=1}^{n+1} \sum_{k=1}^{n} c_{i j} y_{i j k}
$$

subject to

$$
\begin{aligned}
& \sum_{j=1}^{n} \sum_{k=1}^{n} y_{n+1, j, k} \leq n \\
& \sum_{i=1}^{n+1} \sum_{k=1}^{n} y_{i j k} \quad=1 \quad j=1, \ldots, n \\
& \sum_{j=1}^{n+1} \sum_{k=1}^{n} y_{i j k} \quad=1 \quad i=1, \ldots, n \\
& \sum_{j=1}^{n} \sum_{k=1}^{n} y_{n+1, j, k}=\sum_{i=1}^{n} \sum_{k=1}^{n} y_{i, n+1, k} \\
& \sum_{i=1}^{n+1} \sum_{j=1}^{n+1}\left(M_{n+1, j}+M_{j}+M_{i j}+M_{i, n+1}\right) y_{i j k} \leq D_{k} \quad k=1, \ldots, n
\end{aligned}
$$

where the total power of a fully charged battery of vehicle $k$ is $D_{k}$, and $M_{i}$ is the power needed for the trip ending in $i$. Parameter $M_{i j}$ is the power needed for the deadheading distance from trip end $i$ to trip start $j, M_{n+1, j}$ and $M_{i, n+1}$ are what is needed from depot to the first start node and from the last end node to the depot, respectively. 
In this formulation, constraint (14) guarantees that the number of buses intended for use do not exceed the number available. Constraint (17) controls the number of buses leaving the depot, ensuring that the same number of buses return to the depot. Constraint (18) checks that the total power consumption for the chain of trips, per vehicle, not exceed the battery capacity.

\subsubsection{Quick charging}

Using electrical buses with quick charging introduces the need for new input in the VS step. Besides timetables, scheduling constraints and cost specifications, a bus fleet specification is needed indicating used battery technology, number of electrical buses, locations and possibilities to recharge, etc. The new parameters will affect the scheduling constraints as well as cost elements.

The input to the VS step can either include a set of locations (bus stops) where charging can take place (where charging equipment is installed), or a list of potential locations where charging equipment can be installed. Either way, the new model must be able to handle the charging requirements and make sure that the vehicles assigned to each route are able to perform their trips. In the latter case, the model should be formulated so that it chooses the best set of stops where charging equipment should be installed to generate the most efficient solution to the VS.

A feedback mechanism for Step 2 is now even more relevant, since the potentially updated set of charging stops could generate a need for new timetables. The output from VS is not changed (vehicle schedules), but now includes information regarding when and where charging will take place.

In the simplest case which we choose to illustrate here, the actual place where node charging should occur is not chosen, whereas power consumption constraints alone are used to ensure that a vehicle can perform the planned chain of trips. In such a case, we can assume that each vehicle is fully recharged at each charging node it passes from trip start to trip end. Further we also assume that charging is never done during deadheading, and that charging is also available at the depot. Based on these assumption, the power consumption constraints can be formulated as:

$$
\begin{array}{lr}
\sum_{j=1}^{n}\left(M_{n+1, j}+S_{j 1}\right) y_{n+1, j, k} \leq D_{k} & k=1, \ldots, n \\
\sum_{i=1}^{n} S_{j p} y_{i j k} \leq D_{k} & j, k=1, \ldots, n, p=1, \ldots, L_{j} \\
\sum_{j=1}^{n}\left(S_{i, p+1}+M_{i, n+1}\right) y_{i j k} \leq D_{k} & i, k=1, \ldots, n \\
\sum_{i=1}^{n}\left(S_{i, p+1}+M_{i, n+1}\right) y_{i, n+1, k} \leq D_{k} & k=1, \ldots, n
\end{array}
$$


where: $L_{j}$ is the number of nodes that can provide charging during the trip starting at start node $j, S_{j p}$ is the power needed to go from charging node $p-1$ to charging node $p$ in trip starting at node $j$. Parameter $S_{j, p+1}$ is the power needed from the last charging node for a trip starting at node $j$ to the end of the trip.

Under the assumptions made, the battery capacity must be greater than the power needed to go from the depot to the first charging location, from one charging location to another in the same trip, from the last charging location on one trip to the first for another trip, and from the last charging location on the last trip to the depot. This is controlled by constraints (19)-(22).

\subsection{Crew Scheduling (CS)}

The last planning step, Crew Scheduling, is barely affected at all. One could argue that the bus drivers might need to undergo a course on how to operate electric buses, and hence the crew scheduling problem is affected since all bus drivers might not be able to operate all buses. Also, it might be advantageous to plan shift changes (change of drivers) at a stop where charging is possible.

\section{$5 \quad$ Transit operations planning process using electric buses}

Based on our findings in the previous section we have updated the illustration of the transit operations planning process using electric buses. This new version is depicted in Figure 2 including the different aspects and considerations needed when considering a fleet of electric buses. To summarize our findings, we propose the following changes/updates to the public transit planning procedure.

- In NRD the additional inputs of Preference Criteria and Bus Fleet Specifications are needed, and as additional output, we get a list of possible charging stops (as well as information about electrified links, if applicable).

- The TD step needs information on charging times as input, and additionally delivers a list of charging stops.

- In the VS step, a Bus Fleet Specification is additionally needed as input, in order to find feasible schedules with respect to charging constraints/restrictions. The same output is provided as before (vehicle schedules). One might need to go back to TD, and in the worst case, all the way back to NRD, if the set of chosen charging stops does not yield satisfactory results.

These changes are valid for a general planning process, considering all the planning steps, but it is not often that one gets the chance to design a new public transit system from scratch. A more plausible planning situation is to introduce 


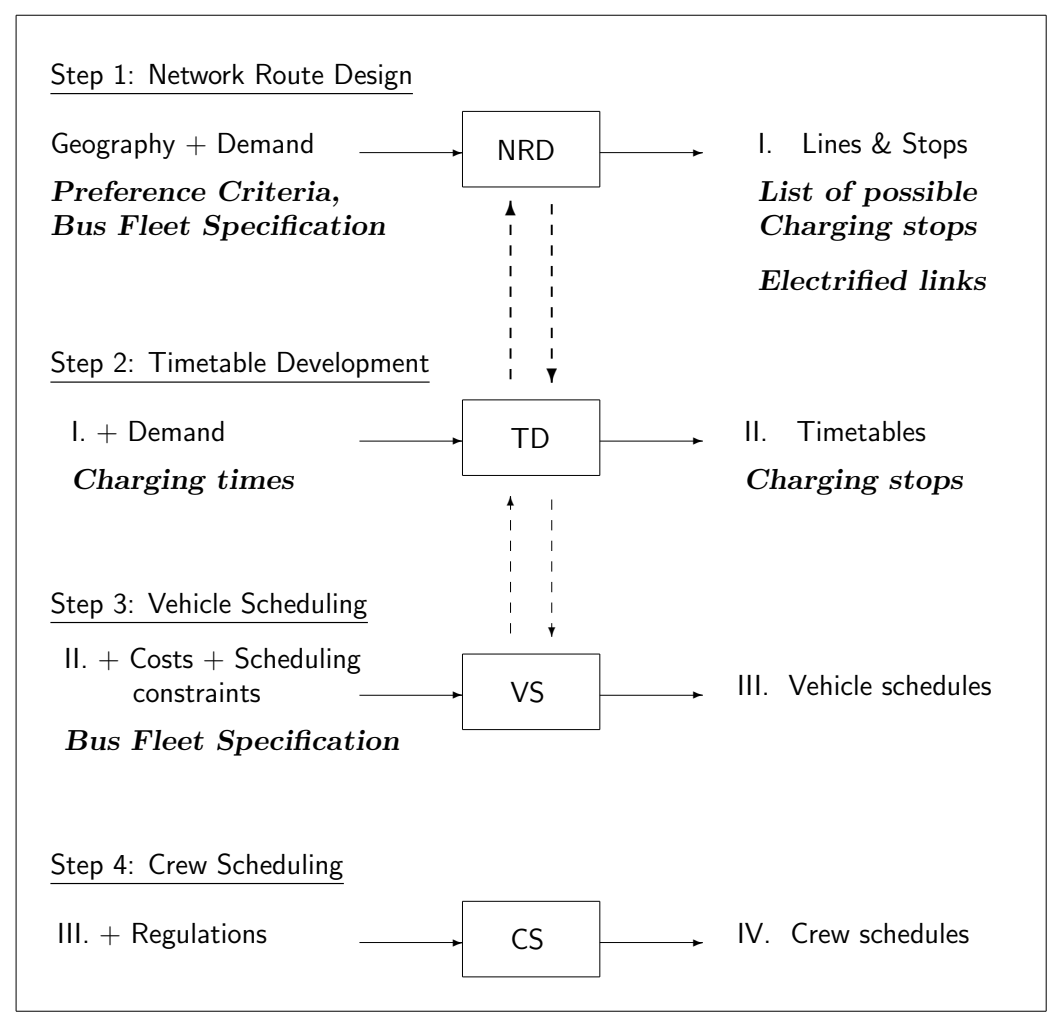

Figure 2: Updated overview of the transit operations planning process using electric buses (with added parts shown in bold italics).

electric buses into an existing transit network, which means that the bus fleet is not necessarily homogeneous. Under such circumstances, other aspects of the planning process, which have not been addressed earlier in this paper, also become relevant.

\section{$5.1 \quad$ Heterogeneous bus fleet}

It is reasonable to argue that, at least during a transition period, the bus fleet will be heterogeneous with a mix of both electric and conventional buses. If only a subset of all buses is electrified, additional planning aspects will be required to design.

\subsubsection{NRD, continuous charging}

Already in the network route design step, when some buses need continuous charging, it may be desirable to prefer electrified bus lines through city centers. To keep installation costs down, this implies a route design where multiple lines share the same route through the center. 


\subsubsection{VS, overnight charging}

When assigning buses to the different lines (routes), where only a subset of the buses are electrified and able to run a full day, electric buses on lines in the city center may be preferable, and should perhaps be required. This could affect interlinings, since non-electric buses may be disqualified for use on some lines. The output from the VS step is however not changed - it is still vehicle schedules that are produced.

\subsection{Long term planning and robustness}

Long term planning becomes relevant under the assumption of a heterogeneous bus fleet with the intention of expanding the number of electrified buses. When designing routes and bus lines, given specifications of the current bus fleet (number of electric buses), should one take into consideration that the number of electric buses will increase and slowly replace conventional buses with combustion engines? Within a few years, with an updated bus fleet, the proposed routes and stops may no longer be optimal or even reasonable.

Robustness is an important aspect in general, but even more so when dealing with electric buses. Timetables and charging schemes (when and where) need to be robust such that if the bus is delayed for some reason, it can be recharged regularly. Also, planning must account for alternative routes during road closure.

\section{A Numerical Real-Life Example}

This section provides a numerical real-life example of how the introduction of electric buses impacts the planning of vehicle schedules. That is, how the number of charging stations and battery capacity will affect the produced vehicle schedules.

We consider a vehicle scheduling problem for electrical vehicles (EV) where the number of vehicles used to cover a set of trips is to be minimized. Each vehicle is assigned to a trip chain, and together all of the trip chains must cover all trips.

A small illustrative real-life example is introduced in Figure 3 based on four bus lines operating in central Norrköping in Sweden. For each line, trips (departures) are considered for both directions, following a given timetable (fixed headways of 10, 20 and 30 minutes). Thus we obtain a total of eight different start and end nodes. Based on the actual distance and travel time for each bus line, from start to end, we derive an estimated value of the energy consumption for each trip. Similarly the energy consumption value for all possible deadheading trips are derived.

We assume the possibility of installing charging equipment at all nodes. In addition, buses have the possibility to charge their batteries for either 5 or 10 minutes. The amount of energy charged into the batteries is not linear with respect to time, that is, a longer charging time is advantageous.

Using different time spans of 2, 3, and 4 hours, three example problems are generated. 


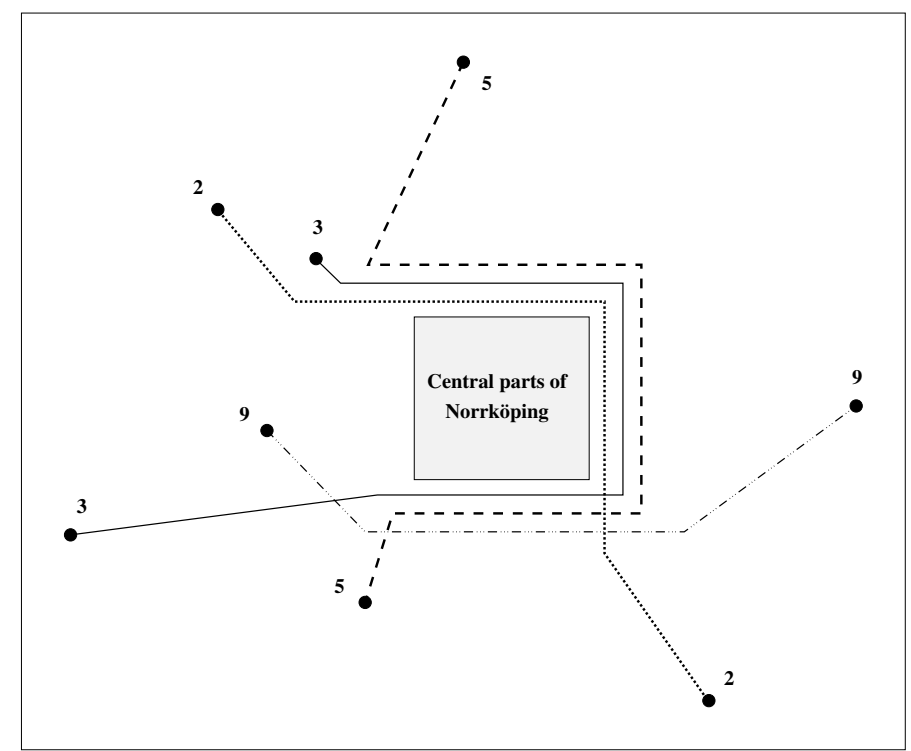

Figure 3: Illustration of the test problem comprised of four bus lines $(2,3,5,9)$ crossing the central of Norrköping.

\subsection{Mathematical Model}

This section formulates and analyzes the vehicle scheduling problem of EVs. The information of the sets, parameters and variables appear in Table 2.

We note that deadheading trips become useful when charging equipment is available only at a subset of the network's nodes.

\subsubsection{The underlying network structure}

The generation of the arc set $\mathcal{A}$ takes care of details required of the problem. For instance, about how to create two consecutive trips with respect to time. An arc between two trips $i$ and $j$, with charging option $k$, exists if the following time constraint is satisfied:

$$
\mathcal{A}=\left\{i, j \in \mathcal{I}, k \in \mathcal{K}: s_{i}+t_{i}+\tau^{k}+T_{i j}+S \leq s_{j}\right\}
$$

That is, the start time of trip $j$ must be larger than the start time of trip $i$ plus the time it takes (i) to perform the trip $t_{i}$, (ii) to charge $\tau^{k}$, (iii) for possible deadheading trip time $T_{i j}$, and (iv) for a buffer time $S$.

An illustration of the network structure appears in Figure 4, with a resulting network of a directed acyclic graph (DAG). Thus, if no considerations are given to a charging activity and to capacity constraints the resulting problem has the integrality property (can be solved as a linear programming (LP)-problem, see e.g. Ahuja, Magnanti, and Orlin (1993)). 
Table 2: Indices, sets, parameters and variables.

\section{Indices and sets}

$\mathcal{K} \quad$ set of charging alternatives $k$

$\mathcal{L}$ set of bus lines $l$

$\mathcal{I} \quad$ set of trips $i$ (all departures for all lines)

$\mathcal{A}$ set of $\operatorname{arcs}(i, j, k)$, possible combinations of trips $i$ and $j$, and charging alternatives $k$

\section{Parameters}

$\lambda$ The weight of deadheading time in the objective function

$N \quad$ Number of available charging stations

$S \quad$ Safety margin between trips (5 min)

$C \quad$ Charging capacity of buses

$C_{\infty} \quad$ Unlimited charging capacity

$q_{i}^{k} \quad$ Energy added by charging option $k$ at charging node $i$

$\tau^{k} \quad$ Time needed to perform charging option $k$

$a_{i} \quad$ Start node for trip $i$

$b_{i} \quad$ Stop node for trip $i$

$s_{i} \quad$ Start time for trip $i$

$d_{i} \quad$ Distance covered by trip $i$

$t_{i} \quad$ Time required to perform trip $i$

$e_{i} \quad$ Energy required by trip $i$

$D_{i j} \quad$ Deadheading distance between $b_{i}$ and $a_{j}$

$T_{i j} \quad$ Time required to go from $b_{i}$ to $a_{j}$

$E_{i j} \quad$ Energy required to go from $b_{i}$ to $a_{j}$

\section{Binary variables}

$x_{i j}^{k} \quad$ equals 1 if a bus performs trip $i$, charge according to $k$, and travel to node $a_{j}$

$w_{l}$ equals 1 if charging is available at the beginning of line $l$

\section{Continuous variables}

$y_{i} \quad$ Energy-level record at node $a_{i}$ after charging

$\bar{y}_{i} \quad$ Energy-level record at node $b_{i}$ after charging

A node (node 0) is also introduced for the depot. This gives that the arc set $\mathcal{A}$ also includes arcs from the depot to the start node of each trip, and from the end node of each trip to the depot. Hence, for a given solution, the number of arcs leaving the depot is equal to the number of buses required. 


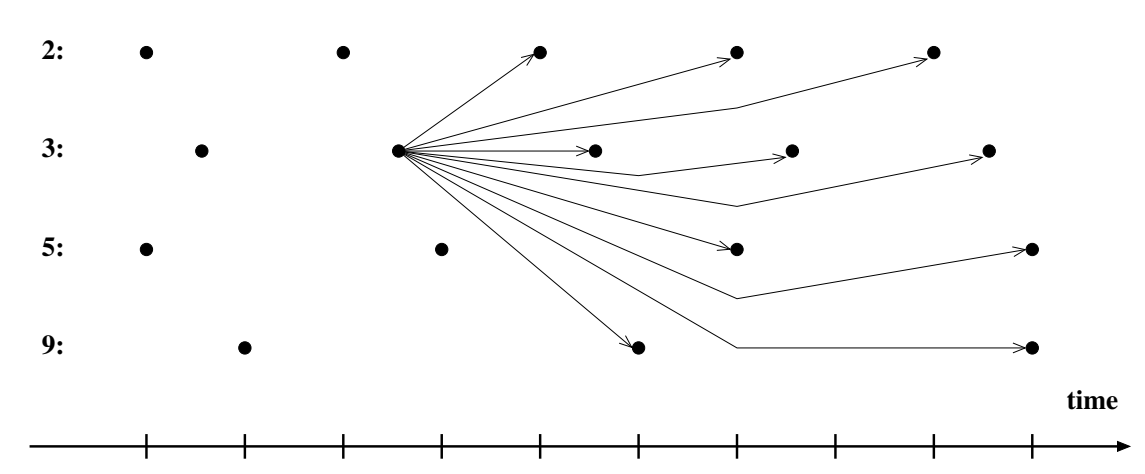

Figure 4: The network structure.

\subsubsection{Energy-level record}

In order to keep track of the energy level for each bus, without introducing busspecific variables, it suffices to introduce an energy-level record for each trip $i \in \mathcal{I}$. This is because of the DAG-structure and the necessity to visit each node exactly once. The model allows for a charging activity both at the end of a trip, node $b_{i}$, and prior to the starting node of the following trip, node $a_{j}$. This is modeled by introducing dummy nodes as is shown in Figure 5.

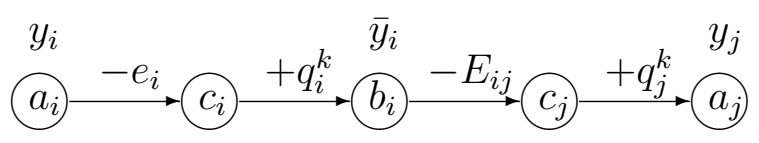

Figure 5: Structure of the arcs.

It is to note that the constraints of the mathematical model limit the energylevel record $y_{i}$ and $\bar{y}_{i}$ to the maximum charging capacity $C$, and also making sure of recording the energy consumption and charging activities.

\subsubsection{Mathematical Model}

A mathematical model for vehicle scheduling of electrical vehicles is described below. The objective function (23) is to minimize the number of buses used (primary objective) as well as minimizing the deadheading distance (secondary objective). Here parameter $\lambda$ is used to control the impacts of the deadheading trips.

Constraints (24) and (25) make sure that each node (trip) is visited exactly once, while constraints (26) and (28) state that in order to perform a trip, a sufficient energy level must be available. Further, constraints (27) and (29) record the energy levels.

Constraints (30) and (31) suppress arcs with charging activities at end nodes where no charging equipment is installed. Finally, constraint (32) limits the number of charging stations to be utilized, and constraints (33)-(35) define all variables. 


$$
\min z=\sum_{(0, j, k) \in \mathcal{A}} x_{0, j}^{k}+\lambda \sum_{(i, j, k) \in \mathcal{A}} T_{i j} x_{i j}^{k}
$$

subject to

$$
\begin{array}{rlrl}
\sum_{(i, j, k) \in \mathcal{A}} x_{i j}^{k} & =1, & & i \in \mathcal{I} \\
\sum_{(i, j, k) \in \mathcal{A}} x_{i j}^{k} & =1, & & j \in \mathcal{I} \\
\sum_{(i, j, k) \in \mathcal{A}} e_{i} x_{i j}^{k} & \leq y_{i}, & & i \in \mathcal{I} \\
\bar{y}_{i} & \leq y_{i}-e_{i}+q_{i}^{k}+C\left(1-x_{i j}^{k}\right), & & (i, j, k) \in \mathcal{A} \\
\sum_{(i, j, k) \in \mathcal{A}} E_{i j} x_{i j}^{k} & \leq \bar{y}_{i}, & & i \in \mathcal{I} \\
y_{j} & \leq \bar{y}_{i}-E_{i j}+q_{j}^{k}+C\left(1-x_{i j}^{k}\right), & & (i, j, k) \in \mathcal{A} \\
\sum_{i j}^{k} & \leq w_{l}, & l \in \mathcal{L}, \\
x_{(i, j, k) \in \mathcal{A}: q_{i}^{k}>0} & & i \in \mathcal{I}: b_{i}=l \\
\sum_{(i, j, k) \in \mathcal{A}: q_{j}^{k}>0} & \leq w_{l}, & l \in \mathcal{L}, \\
\sum_{l \in \mathcal{L}} w_{l} & \leq N & j \in \mathcal{I}: a_{j}=l \\
x_{i j}^{k} & \in\{0,1\}, & & \\
w_{l} & \in\{0,1\}, & & (i, j, k) \in \mathcal{A} \\
y_{i}, \bar{y}_{i} & \leq C, & l \in \mathcal{L} \\
0 \operatorname{lil}_{i j} & & i \in \mathcal{I}
\end{array}
$$

\subsection{Results}

Three example problems were created based on the timetables and frequencies of the four bus lines shown in Figure 3. The example problems are for the consideration of two, three and four hours activities. If assuming that buses have unlimited charging capacity, to be denoted as $C_{\infty}$, the problem can be solved as an LP-problem from which one can find the minimum number of buses required to cover all trips. Table 3 contains the information of these examples.

The safety margin $(S)$ between trips is set to 5 minutes, with a varied charging capacity $C$ throughout the runs. Moreover, each example problem is solved for $N=0,1, \ldots, 8$, the number of available charging stations. The option to charge for 5 minutes provides 10 energy units, and 25 energy units for charging 10 minutes. 
Table 3: Information of the three Example problems. Their names, number of trips, time span and number of buses needed if unlimited charging capacity.

\begin{tabular}{lccc}
\hline Example & No. of trips & Span & $C_{\infty}$ \\
\hline Small & 48 & $(2 \mathrm{~h})$ & 26 \\
Medium & 82 & $(3 \mathrm{~h})$ & 27 \\
Large & 116 & $(4 \mathrm{~h})$ & 27 \\
\hline
\end{tabular}

A value of $\lambda=0.01$ is used in the example problems for putting a higher weight on minimizing the number of buses required. The model is implemented using the software AMPL with CPLEX solver (version 12.5). The calculations of the example problems were performed on a HP DL160 server with two 6-core Intel Xeon CPUs and 72 GB of RAM memory, running Linux, with a time limit of 1 hour for each example problem.

The results of the runs are presented in Table 4 . We note that with a limited capacity $C$ the example problems aren't easy to solve; in this case most of the runs reached their time limit.

Table 4: Results for all problem instances, one row for each number of available charging stations. In each column, the number of buses needed for different charging capacities.

\begin{tabular}{|c|c|c|c|c|c|c|c|c|c|}
\hline \multirow[t]{3}{*}{ Prob. } & \multicolumn{3}{|c|}{ Small (48 trips) } & \multicolumn{3}{|c|}{ Medium (82 trips) } & \multicolumn{3}{|c|}{ Large (116 trips) } \\
\hline & \multicolumn{9}{|c|}{$C$} \\
\hline & 35 & 40 & 45 & 35 & 40 & 45 & 35 & 40 & 45 \\
\hline 0 & 36 & 31 & 27 & 62 & 52 & 40 & 88 & 73 & 56 \\
\hline 1 & 31 & 27 & 27 & 44 & 35 & 31 & 58 & 47 & 39 \\
\hline 2 & 28 & - & 26 & 35 & 31 & 30 & 41 & 34 & 34 \\
\hline 3 & - & & - & 32 & 29 & 29 & 33 & 33 & 30 \\
\hline 4 & & & & 29 & - & 28 & 31 & 30 & 29 \\
\hline 5 & & & & - & & - & 30 & - & - \\
\hline 6 & & & & & & & - & & \\
\hline
\end{tabular}

The results show, not surprisingly, that the higher charging capacity $C$, the lower the number of buses required. A dashed line ("- ") is inserted in Table 4 if by adding charging stations the number of buses is not reduced anymore. However, it is possible that extra charging stations may reduce the time needed for deadheading.

In the three example runs all of the different charging options were used; both 5 and 10 minutes charging time either before or after the deadheading trip or at both ends. 
The resulting trip chains attained were mostly round-trip on the same bus line using a few deadheading trips. However for example problems with a limited number of charging stations ( $N=1$ and $N=2$ ) buses are moving more frequently between the lines so as to visit the nodes with the charging stations.

Certainly, the use of electric buses with a limited charging capacity affects the results of the vehicle scheduling process. This limit increases the number of vehicles required. The modeling and formulation of the optimization problem represent a challenge. Future work can deal with this challenge in the quest for a further strategy to attain an efficient solution.

\section{Conclusion}

A thorough analysis of the public transit operations planning procedure, with respect to the introduction of electric buses and different charging techniques, shows that current planning procedures will not suffice in the future. Multiple new considerations need to be taken into account, and we have presented an updated scheme for the public transit operations planning procedure.

The different charging techniques will influence the planning process in different ways, and to a varying extent. With overnight charging and continuous charging the main challenges are in the network route design step, given the possibility of altering the existing route network, with efficient and optimal changes of the timetabling and vehicle scheduling components. Future research may investigate the impacts of the changes, of the routing and scheduling, on passenger demand and level-of-service provided from the user's perspective.

Furthermore, since electric buses will likely replace conventional buses over the course of many years, planners must decide how to address transitional issues such as heterogeneous bus fleets, and robustness features such as feasible backup lines to use when roads are closed for maintenance. Our collaborating practitioners validate our concerns. The conclusions are, based on their unanimous opinions, that introducing electric buses will definitely affect the current operations planning process. To begin with, infrastructural changes are unavoidable, and it is reasonable to use this opportunity to introduce changes in the design of the network of routes in an optimal manner.

Some concerns are also raised about procurement, with respect to installation and usage of charging equipment, as well as for the possibility of competing companies which somehow have to share this equipment. Future research will have to consider such issues when adjusting the different operations planning steps.

Finally, an illustrative real-life example with four bus lines in Norrköping, Sweden, is formulated and introduced at the last part of work using three problem instances of 48, 82 and 116 bus trips. The main results exhibit the minimum number of vehicles required using different scenarios of charging stations. 


\section{Funding}

This work was supported by the Swedish Energy Agency under Grant 40377-1.

\section{References}

Ahuja, R., Magnanti, T., \& Orlin, J. (1993). Network flows: theory, algorithms, and applications. Upper Saddle River, NJ, USA: Prentice-Hall, Inc.

Bayindir, K., Gözüküçük, M., \& Teke, A. (2011). A comprehensive overview of hybrid electric vehicle: Powertrain configurations, powertrain control techniques and electronic control units. Energy Conversion and Management, 52(2), 1305-1313.

Beltran, B., Carrese, S., Cipriani, E., \& Petrelli, M. (2009). Transit network design with allocation of green vehicles: A genetic algorithm approach. Transportation Research Part C: Emerging Technologies, 17, 475-483.

Brunton, L. (1992, Feb). The trolleybus story. IEE Review, 38(2), 57-61.

Ceder, A. (2016). Public transit planning and operation: Modeling, practice and behavior, second edition. CRC Press.

Ceder, A., Golany, B., \& Tal, O. (2001). Creating bus timetables with maximal synchronization. Transportation Research Part A, 35, 913-928.

Corman, F., D'Ariano, A., \& Hansen, I. (2014). Evaluating disturbance robustness of railway schedules. Journal of Intelligent Transportation Systems, 18(1), 106-120.

Hug, V. (2015, September). Copenhagen trial with $12 \mathrm{~m}$ b.y.d $\mathrm{k} 9$ electric buses. Nordic Electric Bus Initiatives. Gothenburg, Sweden.

Ibarra-Rojas, O., Delgado, F., Giesen, R., \& Muñoz, J. (2015). Planning, operation, and control of bus transport systems: A literature review. Transportation Research Part B: Methodological, 77, 38-75.

Ibarra-Rojas, O., \& Rios-Solis, Y. (2012). Synchronization of bus timetabling. Transportation Research Part B: Methodological, 46, 599-614.

Kliewer, N., Mellouli, T., \& Suhl, L. (2006). A time-space network based exact optimization model for multi-depot bus scheduling. European Journal of Operational Research, 175, 1616-1627.

Li, J. (2014). Transit bus scheduling with limited energy. Transportation Science, 48, 521-539.

Li, J.-Q. (2016). Battery-electric transit bus developments and operations: A review. International Journal of Sustainable Transportation, 10(3), 157-169.

Liu, Z., Shen, J., Wang, H., \& Yang, W. (2007). Regional bus timetabling model with synchronization. Journal of Transportation Systems Engineering and Information Technology, 7(2), 109-112.

Mahmoud, M., Garnett, R., Ferguson, M., \& Kanaroglou, P. (2016). Electric buses: A review of alternative powertrains. Renewable and Sustainable Energy Reviews, 62, 673-684. 
Mohamed, M., Farag, H., El-Taweel, N., \& Ferguson, M. (2017). Simulation of electric buses on a full transit network: Operational feasibility and grid impact analysis. Electric Power Systems Research, 142, 163-175.

Olsson, O., Grauers, A., \& Pettersson, S. (2016, June). Method to analyze cost effectiveness of different electric bus systems. In Proceedings of the EVS29 symposium. Montréal, Québec, Canada.

Parbo, J., Nielsen, O., \& Prato, C. (2014). User perspectives in public transport timetable optimisation. Transportation Research Part C: Emerging Technologies, 48, 269-284.

Perrotta, D., Macedo, J., Rossetti, R., Freire de Sousa, J., Kokkinogenis, Z., Ribeiro, B., \& Afonso, J. (2014). Route planning for electric buses: A case study in oporto. Procedia - Social and Behavioral Sciences, 111, 1004-1014.

Reuer, J., Kliewer, N., \& Wolbeck, L. (2015). The electric vehicle scheduling problem: A study on time-space network based and heuristic solution approaches. Presented at the 13th Conference on Advanced Systems in Public Transport (CASPT).

Saharidis, G., Dimitropoulos, C., \& Skordilis, E. (2014). Minimizing waiting times at transitional nodes for public bus transportation in greece. Operational Research, $14(3), 341-359$.

Živanović, Z., \& Nikolić, Z. (2012). The application of electric drive technologies in city buses. In Z. Stevic (Ed.), New generation of electric vehicles (p. 165-203). Intech.

Wang, H., \& Shen, J. (2007). Heuristic approaches for solving transit vehicle scheduling problem with route and fueling time constraints. Applied Mathematics and Computation, 190, 1237-1249.

Xu, Y., Çolak, S., Kara, E., Moura, S., \& González, M. (2018). Planning for electric vehicle needs by coupling charging profiles with urban mobility. Nature Energy, Advance online publication. doi: 10.1038/s41560-018-0136-x

ZeEUS. (2017). ZeEUS eBus Report \#2 - An updated overview of electric buses in Europe. Technical Report. Retrieved from http://zeeus.eu/publications

Zhu, C., \& Chen, X. (2013). Optimizing battery electric bus transit vehicle scheduling with battery exchanging: Model and case study. Procedia - Social and Behavioral Sciences, 96, 2725-2736.

Zhu, C., Chen, X., \& Xie, J. (2013). Optimization model for locating electric bus charging stations. In Proceedings of the fourth international conference on transportation engineering (icte2013) (p. 1545-1551). 